\title{
Estresse e graduação em Enfermagem: Um estudo com estudantes-trabalhadores
}

\author{
Nursing stress and graduation: A study with student-workers \\ Estrés y graduación en enfermería: Un estudio con estudiantes-trabajadores
}

Recebido: 27/05/2021 | Revisado: 06/06/2021 | Aceito: 12/06/2021 | Publicado: 24/06/2021

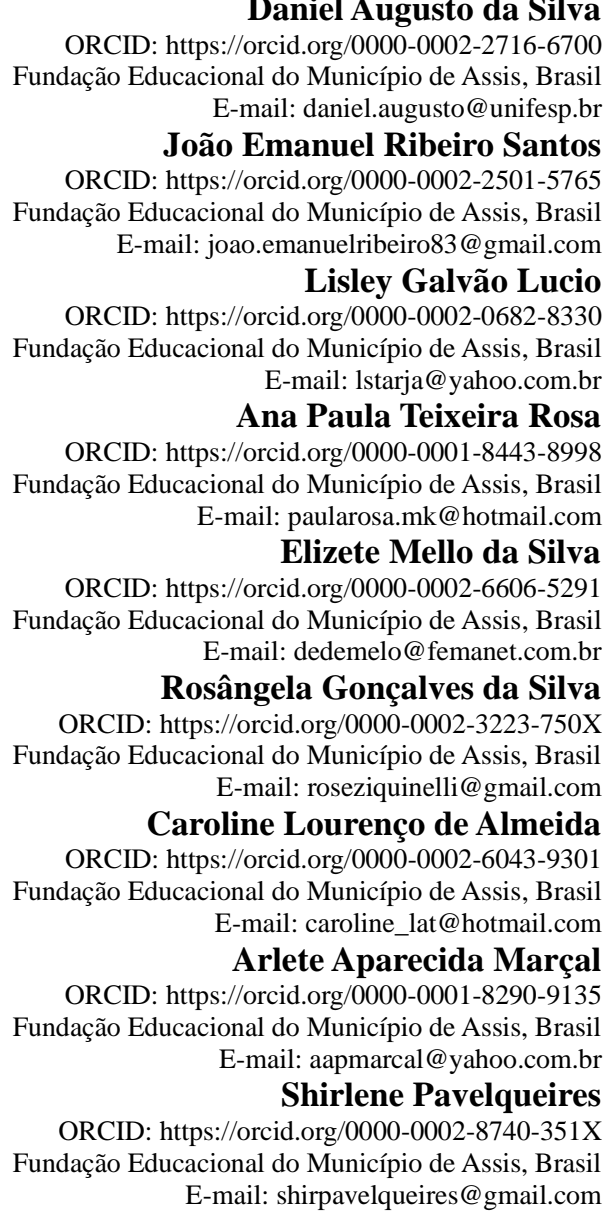

\begin{abstract}
Resumo
Objetivo: identificar a ocorrência do estresse, e vulnerabilidades sociodemográficas, em estudantes de graduação em Enfermagem. Metodologia: Trata-se de estudo quantitativo, transversal, epidemiológico, com 59 estudantes de graduação em Enfermagem de cidade no centro-oeste do estado de São Paulo. Os dados foram coletados entre os meses de setembro e outubro de 2018, por meio de questionário semiestruturado, elaborado pelos autores e Escala de Estresse Percebido. Os dados foram analisados com estatística descritiva e análise inferencial com ANOVA um fator e ANOVA dois fatores fixos, tendo como variável dependente o escore de estresse obtido por meio da Escala de Estresse Percebido, e como preditores as variáveis sociodemográficas. Realizou-se o Teste de igualdade de variância, Levene, e não foi evidenciado diferença estatisticamente significante entre todas as variáveis deste estudo. O nível de significância adotado para as análises deste estudo foi de p $<0,05$. Resultados: Este estudo obteve a participação voluntária de $59(48,0 \%)$ estudantes universitários de graduação em Enfermagem, de 123 (100,0\%) possíveis, que apresentaram média geral de pontuações na Escala de Estresse Percebido igual a 28,2 e moda 27,0. Evidenciou-se diferença estatisticamente significante entre o rastreamento do estresse pela pontuação obtida na aplicação da Escala de Estresse Percebido em estudantes universitários com transtorno mental $(\mathrm{p}=0,020)$, de modo que é possível afirmar que estudantes diagnosticados com transtornos mentais apresentam nível superior de estresse quando comparados a estudantes universitários que não possuem o diagnóstico de transtornos mentais. Considerações finais: Considerando que todas as variáveis apresentaram valor de $\mathrm{p}$ maior que 0,05 , aceita-se a hipótese nula, de forma que não há interação entre o fato de trabalhar e as variáveis estudadas que resultem em percepção diferente de estresse.
\end{abstract}


Palavras-chave: Estudantes de enfermagem; Educação superior; Trabalhadores; Saúde do estudante; Estresse psicológico.

\begin{abstract}
Objective: to identify the occurrence of stress, and sociodemographic vulnerabilities, in undergraduate nursing students. Methodology: This is a quantitative, cross-sectional, epidemiological study, with 59 undergraduate students in Nursing in the city in the central-west of the state of São Paulo. Data were collected between the months of September and October 2018, using a semi-structured questionnaire, prepared by the authors and the Perceived Stress Scale. The data were analyzed with descriptive statistics and inferential analysis with one-way ANOVA and twofactor ANOVA, with the stress score obtained using the Perceived Stress Scale as the dependent variable, and sociodemographic variables as predictors. The Levene Equality of Variance Test was carried out and there was no statistically significant difference between all the variables in this study. The level of significance adopted for the analyzes of this study was $\mathrm{p}<0.05$. Results: This study obtained the voluntary participation of 59 (48.0\%) undergraduate nursing students, of $123(100.0 \%)$ possible, who had a general average of scores on the Perceived Stress Scale equal to 28.2 and fashion 27.0. There was a statistically significant difference between the stress tracking by the score obtained in the application of the Perceived Stress Scale in university students with mental disorder ( $\mathrm{p}=$ 0.020), so that it is possible to state that students diagnosed with mental disorders have a higher level of stress when compared to university students who are not diagnosed with mental disorders. Final considerations: Considering that all variables had a p-value greater than 0.05 , the null hypothesis is accepted, so that there is no interaction between the fact of working and the variables studied that result in a different perception of stress.
\end{abstract}

Keywords: Students, Nursing; Higher education; Workers; Student health; Psychological stress.

\title{
Resumen
}

Objetivo: identificar la ocurrencia de estrés y vulnerabilidades sociodemográficas en estudiantes de licenciatura en enfermería. Metodología: Se trata de un estudio epidemiológico, cuantitativo, transversal, con 59 estudiantes de pregrado en Enfermería de la ciudad del centro-oeste del estado de São Paulo. Los datos se recolectaron entre los meses de septiembre y octubre de 2018, mediante un cuestionario semiestructurado, elaborado por los autores y la Escala de Estrés Percibido. Los datos se analizaron con estadística descriptiva y análisis inferencial con ANOVA de una vía y ANOVA de dos factores, con el puntaje de estrés obtenido utilizando la Escala de Estrés Percibido como variable dependiente y variables sociodemográficas como predictoras. Se realizó la prueba de igualdad de varianza de Levene y no hubo diferencia estadísticamente significativa entre todas las variables de este estudio. El nivel de significancia adoptado para los análisis de este estudio fue $\mathrm{p}<0.05$. Resultados: Este estudio obtuvo la participación voluntaria de 59 (48.0\%) estudiantes de licenciatura en enfermería, de 123 (100.0\%) posibles, quienes tuvieron un promedio general de puntajes en la Escala de Estrés Percibido igual a 28.2 y moda 27.0. Hubo diferencia estadísticamente significativa entre el seguimiento del estrés por la puntuación obtenida en la aplicación de la Escala de Estrés Percibido en estudiantes universitarios con trastornos mentales $(\mathrm{p}=0,020)$, por lo que es posible afirmar que los estudiantes diagnosticados con trastornos mentales tienen un mayor nivel de estrés en comparación con los estudiantes universitarios que no están diagnosticados con trastornos mentales. Consideraciones finales: Considerando que todas las variables tuvieron un p-valor mayor a 0.05 , se acepta la hipótesis nula, por lo que no existe interacción entre el hecho de trabajar y las variables estudiadas que resulten en una percepción diferente del estrés.

Palabras clave: Estudiantes de enfermería; Educación universitaria; Trabajadores; Salud estudiantil; Estrés psicológico.

\section{Introdução}

As ações de expansão de vagas em instituições de ensino superior permitiram o acesso às universidades para jovens adultos, indivíduos que já trabalham e também classes sociais menos favorecidas, contudo, deve-se salientar que esse processo de expansão ocorre em instituições privadas, que exigem o custeio dos estudos, e gera estudantes-trabalhadores (Santos, Loiola \& Santos, 2018; Souza \& Vazquez, 2015; Souza 2017).

Destaca-se que a condição de ser estudante-trabalhador é produto da acreditação da ascensão social e melhoria das condições de vida por meio da conclusão do ensino superior, todavia, realiza-se uma crítica quanto ao caminho equivocado para este objetivo quando se observa, na atualidade, a reserva de mercado devido ausência de vagas de emprego a todos os portadores de diploma de nível superior (Silva \& Marcolan, 2015).

Aos esforços empregados no custeio do ensino superior, e o enfrentamento do desafio de estudar e trabalhar por alguns anos, unem-se realidades fisiológicas, sociais, cognitivas e emocionais inesperadas, respectivas a vida acadêmica, que 
apresentam potencial de influenciar comportamentos. Entre as situações, salienta-se a adaptação ao novo ambiente, novas obrigações, novas modalidades de avaliações, preocupações com o futuro profissional e inserção no mercado de trabalho, conflitos com colegas e outros profissionais, gerenciamento de tempo entre estudo e vida familiar, separação do núcleo familiar, cobrança por parte da família e auto cobrança, entre outros (Silva, 2019; Silva, Pereira Junior, Gomes \& Cardoso, 2019; Gomes, Silva \& Aranzate, 2018; Ribeiro, Bragiola, Eid \& Pompeo, 2020; Mussi, Pires, Carneiro, Costa, Ribeiro \& Santos, 2019).

No reconhecimento dessas situações, o organismo tenta realizar adaptações, e quando não consegue, desenvolve-se o estresse patológico, como resultado da vulnerabilidade frente a uma série de mudanças impostas e necessidades de adaptação (Silva, 2019; Silva, Pereira Junior, Gomes \& Cardoso, 2019; Gomes, Silva \& Aranzate, 2018; Ribeiro, Bragiola, Eid \& Pompeo, 2020). Em outras palavras, o estresse pode ser entendido como "o peso da sociedade sore o indivíduo" (Vieira, Russo, 2019).

$\mathrm{O}$ estresse é desenvolvido em quatro fases: alerta/alarme, resistência, quase-exaustão e exaustão. Na fase de alerta/alarme, a reação de luta ou fuga possui relação com a produção de adrenalina, para preservação da própria vida. Na resistência, as reservas fisiológicas foram mobilizadas para aumentar a capacidade de resistência acima do normal, de forma que a busca pelo reequilíbrio emana de energia considerável, e gera desgaste generalizado. Na fase de quase-exaustão, muitos sintomas podem ser percebidos, de forma intermitente, com oscilação entre momentos de bem-estar e tranquilidade e momentos de desconforto, ansiedade e cansaço. Há diminuição de defesas e resistências. Na última fase, a exaustão, observase sensação de consumo total da energia, pelo indivíduo, de forma que esta passa a depender de reposição de energia adaptativa, como os medicamentos (Lipp 2008; Lipp 2004; Cardoso, Gomes, Pereira Junior \& Silva, 2019).

Nesta perspectiva, enfatiza-se a importância de elaborar ações de promoção e prevenção à saúde no contexto universitário, com foco no estresse, que estejam alinhadas a realidade vivenciada, conhecida através da avaliação e identificação do nível deste.

Dessa forma, este estudo teve por objetivo identificar a ocorrência do estresse, e vulnerabilidades sociodemográficas, em estudantes de graduação em Enfermagem.

\section{Metodologia}

Trata-se de estudo quantitativo, transversal, epidemiológico, com 59 estudantes de graduação em Enfermagem, matriculados em instituição de ensino superior de cidade no centro-oeste do estado de São Paulo.

Optou-se por um modelo de amostragem não probabilística por conveniência, de modo que todos os alunos que atenderam aos critérios de inclusão foram considerados a população do estudo. Os critérios de inclusão compreenderam ser aluno regularmente matriculado na instituição, estar presente na sala de aula no dia elegido para coleta dos dados e consentir com a participação voluntária.

Os dados foram coletados entre os meses de setembro e outubro de 2018, por meio de questionário semiestruturado, elaborado pelos autores, para caracterização dos participantes quanto às variáveis sociodemográficas e aplicação da Escala de Estresse Percebido, composta por 14 perguntas com respostas em escala tipo Likert de cinco pontos e escore final que possibilita pontuação entre zero e 56 pontos (Luft, Sanches, Mazo, \& Andrade, 2007). Os participantes deste estudo dedicaram em média dez minutos para responder aos questionários.

Os dados foram analisados com estatística descritiva e análise inferencial com ANOVA um fator e ANOVA dois fatores fixos, tendo como variável dependente o escore de estresse obtido por meio da Escala de Estresse Percebido, e como preditores as variáveis sociodemográficas. Realizou-se o Teste de igualdade de variância, Levene, e não foi evidenciado diferença estatisticamente significante entre todas as variáveis deste estudo. O nível de significância adotado para as análises 
deste estudo foi de $\mathrm{p}<0,05$.

Todos os participantes deste estudo assinaram o Termo de Consentimento Livre e Esclarecido, concordando a participação e somente após responderam ao questionário respectivo a esta pesquisa, respeitando a legislação específica para pesquisas com seres humanos, Resolução 466/2012.

O projeto foi submetido ao Comitê de Ética em Pesquisa da Fundação Educacional do Município de Assis (FEMA), sob CAAE 92564218.9.0000.8547 e obteve aprovação sob parecer nº 2.895.995, de 14 de setembro de 2018.

\section{Resultados}

Este estudo obteve a participação voluntária de 59 (48,0\%) estudantes universitários de graduação em Enfermagem, de $123(100,0 \%)$ possíveis, que apresentaram média geral de pontuações na Escala de Estresse Percebido igual a 28,2 e moda 27,0 .

A variação de pontuação foi entre 2,0 e 47,0, cabendo ressaltar que a possibilidade de pontuação conforme o este instrumento varia de 0,0 a 56,0 pontos, o que, por sua vez, possibilita a afirmação de que todos os participantes revelaram a vivência do estresse, em diferentes níveis de intensidade (Figura 1).

Figura 1. Boxplot da avaliação do estresse percebido em estudantes universitários de Enfermagem ( $\mathrm{n}=59)$. Assis (SP), Brasil, 2021.

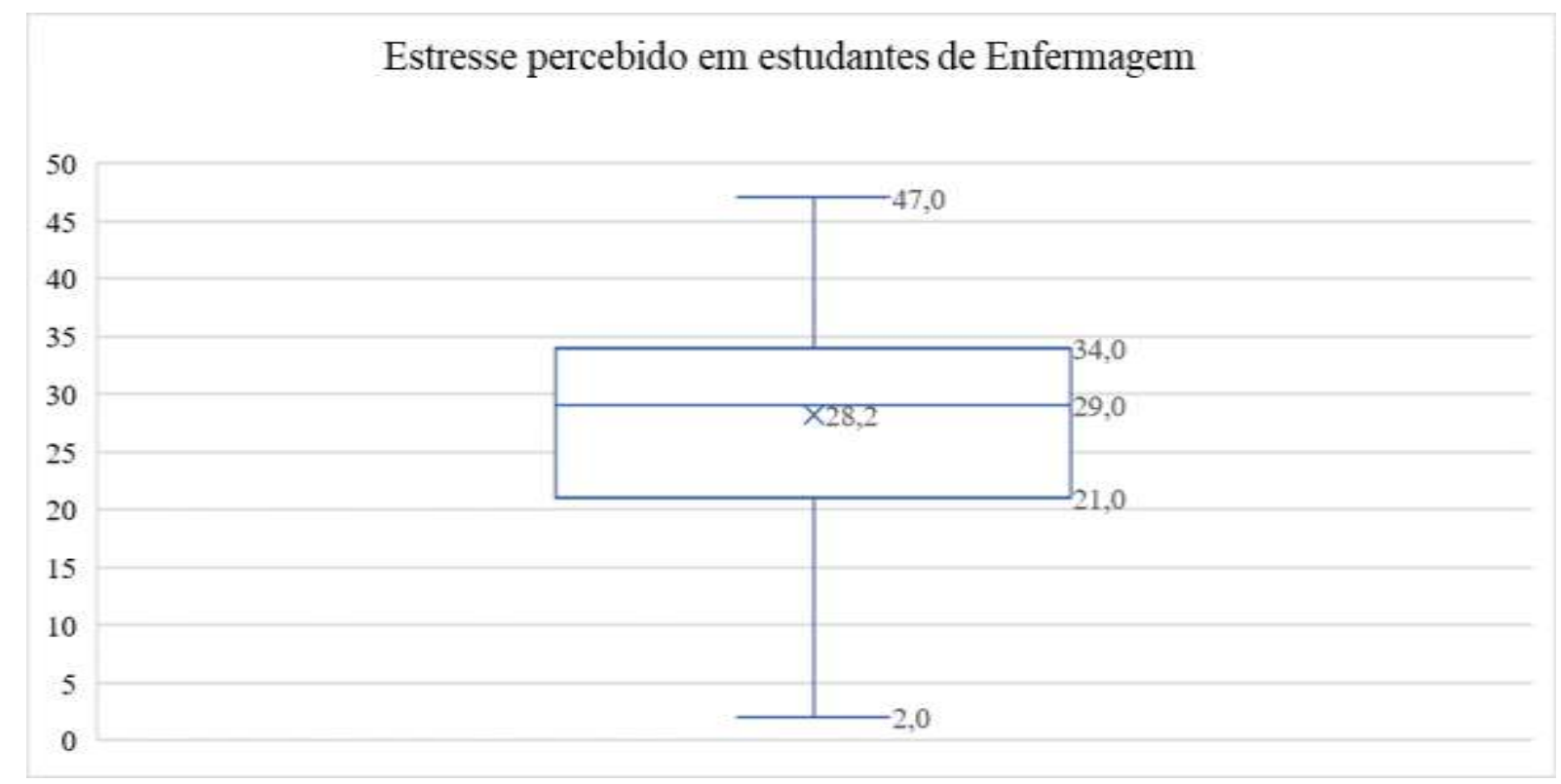

Fonte: Autores.

$\mathrm{Na}$ caracterização dos participantes, associados à avaliação do estresse, observa-se maior participação de mulheres $(91,5 \%)$, nas quais a média de estresse foi maior $(28,76)$ do que em homens $(22,40)$. Outras informações sobre a caracterização sociodemográfica dos participantes e a avaliação do estresse estão descritas na Tabela 1. 
Tabela 1. Associação entre as variáveis sociodemográficas e o rastreamento para estresse percebido em estudantes universitários de Enfermagem $(\mathrm{n}=59)$. Assis $(\mathrm{SP})$, Brasil, 2021.

\begin{tabular}{|c|c|c|c|c|c|c|}
\hline \multirow[t]{2}{*}{ Variáveis } & \multirow[t]{2}{*}{ n $(\%)$} & \multicolumn{4}{|c|}{ Escala de Estresse Percebido } & \multirow[t]{2}{*}{ p-Valor* } \\
\hline & & Variação & Média & Mediana & Moda & \\
\hline SEXO & & & & & & 0,124 \\
\hline Feminino & $54(91,5)$ & $2-47$ & 28,76 & 29,5 & - & \\
\hline Masculino & $5(8,5)$ & $12-38$ & 22,40 & 15,0 & 27 & \\
\hline FAIXA ETÁRIA & & & & & & 0,151 \\
\hline Jovem - até 19 anos & $16(27,1)$ & $18-47$ & 30,94 & 33,5 & 34 & \\
\hline Adulto - 20 a 50 anos & $43(72,9)$ & $2-47$ & 27,21 & 28,0 & 27 & \\
\hline ORIENTAÇÃO SEXUAL & & & & & & 0,099 \\
\hline Heterossexual & $56(94,9)$ & $2-47$ & 27,91 & 29,5 & 27 & \\
\hline Homossexual & $2(3,4)$ & $27-28$ & 27,50 & 27,5 & - & \\
\hline Bissexual & $1(1,7)$ & 47 & 47,00 & - & - & \\
\hline FILHOS & & & & & & 0,208 \\
\hline Não & $48(84,1)$ & $14-47$ & 28,92 & 28,5 & 27 & \\
\hline Sim & $11(18,6)$ & $2-39$ & 25,18 & 31,0 & 38 & \\
\hline RELIGIÃO & & & & & & 0,298 \\
\hline Sim & $55(93,2)$ & $2-47$ & 28,55 & 30,0 & 27 & \\
\hline Não & $4(6,8)$ & $12-38$ & 23,75 & 22,5 & - & \\
\hline PATOLOGIA FÍSICA & & & & & & 0,449 \\
\hline Não & $53(89,8)$ & $2-47$ & 27,92 & 28,0 & 27 & \\
\hline Sim & $6(10,2)$ & $12-38$ & 30,83 & 35,0 & - & \\
\hline TRANSTORNO MENTAL & & & & & & $\mathbf{0 , 0 2 0}$ \\
\hline Não & $51(86,4)$ & $2-45$ & 27,18 & 27,0 & 27 & \\
\hline Sim & $8(13,6)$ & $18-47$ & 34,88 & 36,0 & 38 & \\
\hline ESTUDANTE TRABALHADOR & & & & & & 0,507 \\
\hline Sim & $34(57,6)$ & $2-45$ & 27,56 & 29,5 & 21 & \\
\hline Não & $25(42,4)$ & $18-47$ & 29,12 & 27,0 & 27 & \\
\hline
\end{tabular}

* ANOVA 1 fator

Fonte: Autores.

Evidenciou-se diferença estatisticamente significante entre o rastreamento do estresse pela pontuação obtida na aplicação da Escala de Estresse Percebido em estudantes universitários com transtorno mental ( $\mathrm{p}=0,020)$, de modo que é possível afirmar que estudantes diagnosticados com transtornos mentais apresentam nível superior de estresse quando comparados a estudantes universitários que não possuem o diagnóstico de transtornos mentais.

Ainda, destaca-se que, em relação à orientação sexual, há tendência para diferença de comportamento entre os grupos (heterossexuais, homossexuais e bissexuais), com $\mathrm{p}=0,099$.

No seguimento da apresentação e análise dos resultados, conforme objetivo deste estudo, os participantes foram classificados como trabalhadores e não trabalhadores, e estes grupos apresentaram média de pontuações na Escala de Estresse Percebido igual a 27,6 e 29,1 respectivamente. A Figura 2 demonstra as diferenças de avaliação do estresse entre esses grupos. 
Figura 2. Boxplot de comparação na avaliação do estresse percebido em estudantes universitários de Enfermagem trabalhadores e não trabalhadores $(\mathrm{n}=59)$. Assis $(\mathrm{SP})$, Brasil, 2021.

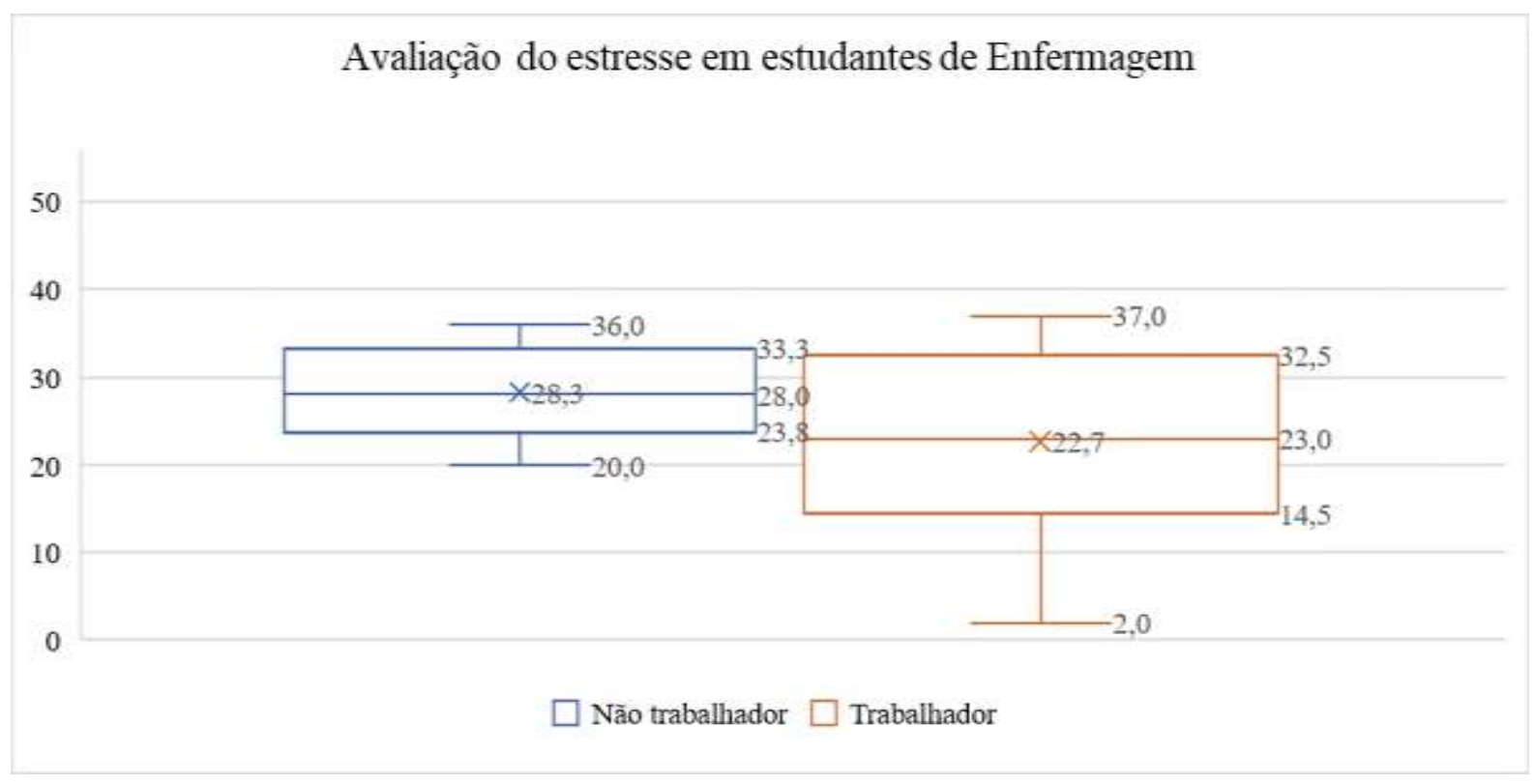

Fonte: Autores.

$\mathrm{Na}$ análise do estresse como resultado da interação entre as variáveis sociodemográficas deste estudo e o fato de ser um estudante trabalhador, a Tabela 2 expõe os dados.

Considerando que todas as variáveis apresentaram valor de p maior que 0,05, aceita-se a hipótese nula, de forma que não há interação entre o fato de trabalhar e as variáveis estudadas que resultem em percepção diferente de estresse.

Ressalta-se que, em análise descritiva, verificam-se as médias de pontuação de estresse, na maioria das vezes, menor em estudantes trabalhadores quando comparadas a estudantes não trabalhadores. 
Tabela 2. Comparação entre estudantes universitários de Enfermagem que exercem atividade remunerada e os que se dedicam integralmente aos estudos, em relação as variáveis sociodemográficas e o rastreamento para estresse percebido $(\mathrm{n}=59)$. Assis (SP), Brasil, 2021.

\begin{tabular}{|c|c|c|c|c|c|c|c|}
\hline \multirow[b]{2}{*}{ Variáveis (n; \%) } & \multicolumn{3}{|c|}{ Estudante trabalhador } & \multicolumn{3}{|c|}{ Estudante não trabalhador } & \multirow{2}{*}{$\begin{array}{l}\text { p- } \\
\text { Valor } * *\end{array}$} \\
\hline & $\begin{array}{c}\mathrm{n} \\
(\%)\end{array}$ & $\begin{array}{l}\text { Média } \\
\text { EPS* }\end{array}$ & $\begin{array}{c}\text { Variação } \\
\text { EPS* }\end{array}$ & $\begin{array}{c}\mathrm{n} \\
(\%)\end{array}$ & $\begin{array}{l}\text { Média } \\
\text { EPS* }\end{array}$ & $\begin{array}{c}\text { Variação } \\
\text { EPS* }\end{array}$ & \\
\hline \multicolumn{8}{|l|}{ SEXO } \\
\hline $\begin{array}{l}\text { Feminino } \\
(54 ; 91,5)\end{array}$ & $\begin{array}{r}29 \\
(53,7) \\
\end{array}$ & 28,4 & $2-45$ & $\begin{array}{r}25 \\
(46,3) \\
\end{array}$ & 29,1 & $18-47$ & \\
\hline $\begin{array}{l}\text { Masculino } \\
(5 ; 8,5)\end{array}$ & $\begin{array}{r}5 \\
(100,0)\end{array}$ & 22,4 & $12-38$ & $0(0,0)$ & - & - & \\
\hline FAIXA ETÁRIA & & & & & & & 0,486 \\
\hline $\begin{array}{l}\text { Jovem - até } 19 \text { anos } \\
(16 ; 27,1)\end{array}$ & $8(50,0)$ & 31,6 & $18-45$ & $8(50,0)$ & 30,3 & $20-47$ & \\
\hline $\begin{array}{l}\text { Adulto - } 20 \text { a } 50 \text { anos } \\
(43 ; 72,9)\end{array}$ & $\begin{array}{r}26 \\
(60,5) \\
\end{array}$ & 26,3 & $2-39$ & $\begin{array}{r}17 \\
(39,5) \\
\end{array}$ & 28,6 & $18-47$ & \\
\hline ORIENTAÇÃO SEXUAL & & & & & & & 0,882 \\
\hline $\begin{array}{l}\text { Heterossexual } \\
(56 ; 94,9)\end{array}$ & $\begin{array}{r}33 \\
(58,9)\end{array}$ & 27,5 & $2-45$ & $\begin{array}{r}23 \\
(41,1)\end{array}$ & 48,4 & $18-47$ & \\
\hline $\begin{array}{l}\text { Homossexual } \\
(2 ; 3,4)\end{array}$ & $1(50,0)$ & 28,0 & - & $1(50,0)$ & 27,0 & - & \\
\hline $\begin{array}{l}\text { Bissexual } \\
(1 ; 1,7)\end{array}$ & 0 & - & - & $1(10,0)$ & 47,0 & - & \\
\hline FILHOS & & & & & & & 0,137 \\
\hline $\begin{array}{l}\text { Não } \\
(48 ; 84,1)\end{array}$ & $\begin{array}{r}28 \\
(58,3)\end{array}$ & 28,9 & $14-45$ & $\begin{array}{r}20 \\
(41,7) \\
\end{array}$ & 28,9 & $18-47$ & \\
\hline $\begin{array}{l}\text { Sim } \\
(11 ; 18,6)\end{array}$ & $\begin{array}{r}6 \\
(54,5)\end{array}$ & 21,2 & $2-39$ & $5(45,5)$ & 30 & $18-38$ & \\
\hline RELIGIÃO & & & & & & & 0,630 \\
\hline $\begin{array}{l}\text { Sim } \\
(55 ; 93,2)\end{array}$ & $\begin{array}{r}32 \\
(58,2) \\
\end{array}$ & 27,7 & $2-45$ & $\begin{array}{r}23 \\
(41,8) \\
\end{array}$ & 29,7 & $18-47$ & \\
\hline $\begin{array}{l}\text { Não } \\
(4 ; 6,8)\end{array}$ & $\begin{array}{r}2 \\
(50,0)\end{array}$ & 25,0 & $12-38$ & $2(50,0)$ & 22,5 & $18-27$ & \\
\hline PATOLOGIA FÍSICA & & & & & & & 0,413 \\
\hline $\begin{array}{l}\text { Não } \\
(53 ; 89,8)\end{array}$ & $\begin{array}{r}30 \\
(56,6) \\
\end{array}$ & 27,5 & $2-45$ & $\begin{array}{r}23 \\
(43,4) \\
\end{array}$ & 28,5 & $18-47$ & \\
\hline $\begin{array}{l}\text { Sim } \\
(6 ; 10,2)\end{array}$ & $\begin{array}{r}4 \\
(66,7)\end{array}$ & 28,3 & $12-37$ & $2(33,3)$ & 36,0 & $34-38$ & \\
\hline $\begin{array}{l}\text { TRANSTORNO } \\
\text { MENTAL }\end{array}$ & & & & & & & 0,713 \\
\hline $\begin{array}{l}\text { Não } \\
(51 ; 86,4)\end{array}$ & $\begin{array}{r}29 \\
(56,9) \\
\end{array}$ & 26,6 & $2-45$ & $\begin{array}{r}22 \\
(43,1) \\
\end{array}$ & 28,0 & $18-38$ & \\
\hline $\begin{array}{l}\text { Sim } \\
(8 ; 13,6)\end{array}$ & $\begin{array}{r}5 \\
(62,5) \\
\end{array}$ & 33,4 & $28-38$ & $3(37,5)$ & 37,3 & $18-47$ & \\
\hline
\end{tabular}

* Escala de Estresse Percebido

**ANOVA 2 fatores fixos

Fonte: Autores.

\section{Discussão}

Buscando adaptação e sobrevivência em decorrência de situações estressoras, respostas fisiológicas são observadas, como o aumento da concentração de glicose plasmática, o aumento da frequência cardíaca e o aumento de fluxo sanguíneo para os músculos, de forma que o ajuste das funções fisiológicas possa resultar nas reações de fuga ou luta (Rocha, 2018).

As situações estressoras, como também o tipo de resposta de cada pessoa, dependem não somente da magnitude e frequência dos acontecimentos de vida, mas da conjunção de fatores ambientais e genéticos (Margis, 2003).

É neste contexto do entendimento sobre o estresse e os fatores que podem estar envolvidos em seu desenvolvimento 
que os dados deste estudo são apresentados.

Entre os participantes deste estudo, considerável maioria de estudantes de graduação em Enfermagem era do sexo feminino (91,5\%), uma situação histórica na Enfermagem, com 86,2\% dos profissionais enfermeiros do sexo feminino, segundo perfil da Enfermagem no Brasil publicado em 2017 pelo Conselho da categoria (Conselho Federal de Enfermagem, 2017).

A análise da avaliação do estresse revelou maior variação de pontuação entre mulheres ( 2 a 47) do que entre os homens (12 a 38), contudo, a média de pontuação foi maior para as mulheres (28,76). Esse resultado sugere que as mulheres vivenciem o estresse com maior intensidade do que os homens.

A essa questão, pesquisas internacionais realizadas na Coreia, na Austrália e na Inglaterra apresentam maior prevalência de estresse percebido no sexo feminino, comparado com o sexo masculino. No Brasil a prevalência também é dominante no sexo feminino. Inúmeros fatores de risco podem ser apontados para essa situação, todavia, enfatiza-se a necessidade de considerar as características regionais e a maior espontaneidade ao admitir o estresse quando comparadas aos homens (Cardoso, Gomes, Pereira Junior \& Silva, 2019).

A análise das demais variáveis revelou maior média de estresse em estudantes jovens (30,94), quando comparados aos adultos $(27,21)$; estudantes sem filhos $(28,92)$; com religião declarada $(28,55)$; com patologia física $(30,83)$; e com transtorno mental $(34,88)$.

Conforme os objetivos deste estudo, focaremos na análise das pontuações para o rastreamento de estresse percebido com a comparação de estudantes trabalhadores e não trabalhadores.

Obteve-se que os estudantes não trabalhadores apresentaram maior média de estresse $(29,12)$ frente aos estudantes trabalhadores (27,56), mesmo que sem significância estatística ( $\mathrm{p}=0,507)$.

Resultado semelhante ao de pesquisa realizada em outro município do interior paulista que identificou a posição de trabalhador e ser trabalhador na área da saúde com menor chance de desenvolver estresse (Preto, Souza, Sousa, Fernandes, Pereira, \& Cardoso, 2020).

Para a faixa etária, estudantes jovens trabalhadores revelaram maior média de estresse do que estudantes jovens não trabalhadores (31,6 e 30,3 respectivamente). Enquanto que aos adultos houve inversão, com maior média de estresse para não trabalhadores comparados a adultos trabalhadores (28,6 e 26,3 respectivamente).

A entrada para o ensino superior é um fator de risco para o desenvolvimento do estresse, pois os estudantes precisam passar por inúmeras transformações e adaptações, tornando-os mais susceptíveis às grandes possibilidades estressoras no decorrer de suas formações, trazendo dificuldades e até sofrimento por causa das exigências do novo ambiente com as novas realidades (Hirsch, 2018).

Contudo, neste estudo observamos a diferença de afetação em estudantes trabalhadores e não trabalhadores conforme a faixa etária. A afetação foi maior em jovens estudantes trabalhadores, e foi maior em adultos não trabalhadores.

Sugere-se que esta condição se refere a adaptação as mudanças, com jovens deixando a fase da adolescência e agregando responsabilidades, que aqui acumulam o emprego e os estudos. Ao utilizar a palavra acúmulo, buscamos diferenciar da palavra conciliação, pois na verdade não há conciliação entre trabalho e estudo, mas a conciliação limita-se a conciliação dos horários, de forma que possibilita o acúmulo de tarefas. Na realidade, o trabalho atrapalha os estudos, e os estudos atrapalham o trabalho. $\mathrm{Na}$ instituição de ensino, as atividades propostas exigem dedicação de tempo que os estudantes trabalhadores não têm, e, nos postos de trabalho, as condições impostas pelo capitalismo globalizado e neoliberal culminam na ausência de direitos - a precarização (Siqueira, 2007).

Aos adultos, a falta do emprego é geradora de sofrimento e impacto físico, psíquico e social. O desempregado passa a desenvolver sentimentos como insegurança e desconfiança (Silva \& Marcolan, 2015; Oliveira, Godoy \& Fogaça, 2019; 
Campos, Zanini \& Castro, 2013).

$\mathrm{Na}$ associação entre variáveis sociodemográficas e o rastreamento do estresse em estudantes universitários, evidenciou-se diferença estatisticamente significante em estudantes universitários com transtorno mental $(\mathrm{p}=0,020)$,

Pessoas com transtornos mentais estão vulneráveis. O sofrimento vai além dos relacionados aos sinais e sintomas do transtorno, e compreendem, também, a exclusão, a rejeição, a discriminação e a estigmatização, e, por vezes, a pessoa com transtorno mental passa a desenvolver a acreditar na sua incapacidade, imposta pelo estigma social, e desenvolve o auto estigma (Cassiano, Marcolan, \& Silva, 2019).

\section{Considerações Finais}

Este estudo obteve a participação voluntária de 59 (48,0\%) estudantes universitários de graduação em Enfermagem, de $123(100,0 \%)$ possíveis, que apresentaram média geral de pontuações na Escala de Estresse Percebido igual a 28,2 e moda 27,0 .

Evidenciou-se diferença estatisticamente significante entre o rastreamento do estresse pela pontuação obtida na aplicação da Escala de Estresse Percebido em estudantes universitários com transtorno mental ( $\mathrm{p}=0,020)$, de modo que é possível afirmar que estudantes diagnosticados com transtornos mentais apresentam nível superior de estresse quando comparados a estudantes universitários que não possuem o diagnóstico de transtornos mentais.

Ainda, destaca-se que, em relação a orientação sexual, há tendência para diferença de comportamento entre os grupos (heterossexuais, homossexuais e bissexuais), com maior pontuação média de estresse para heterossexuais ( $\mathrm{p}=0,099$ ).

Considerando que todas as variáveis apresentaram valor de p maior que 0,05 , aceita-se a hipótese nula, de forma que não há interação entre o fato de trabalhar e as variáveis estudadas que resultem em percepção diferente de estresse.

Ressalta-se que, em análise descritiva, verifica-se as médias de pontuação de estresse, na maioria das vezes, menor em estudantes trabalhadores quando comparadas a estudantes não trabalhadores.

\section{Referências}

Campos, D. C., Zanini, D. S., \& Castro, L. G. (2013). Desemprego e estresse: tipos de problemas vivenciados e relatados pelos desempregados. Fragmentos de Cultura, 23(3), 379-387. http://seer.pucgoias.edu.br/index.php/fragmentos/article/view/2957/1810

Cardoso, J. V., Gomes, C. F. M., Pereira Junior, R. J., \& Silva, D. A. (2019). Estresse em estudantes universitários: uma abordagem epidemiológica. Rev enferm UFPE on line, 13, e241547. https://doi.org/10.5205/1981-8963.2019.241547

Cassiano, A. P. C., Marcolan, J. F., \& Silva, D. A. (2019). Atenção primária à saúde: estigma a indivíduos com transtornos mentais. Rev enferm UFPE on line, 13, e239668. https://doi.org/10.5205/1981-8963.2019.239668

Conselho Federal de Enfermagem. (2017). Relatório final da Pesquisa Perfil da Enfermagem no Brasil - Fiocruz, Rio de Janeiro.

Gomes, A. S., Silva, G. V., \& Aranzate, R R. (2018). A evolução do estresse e a fase de quase-exaustão como fator desencadeador da depressão. Revista Dissertar, 30(1), 131-52. https://doi.org/10.24119/16760867ed114236 4

Hirsch, C. D., Barlem, E. L. D., Almeida, L. K. D., Tomaschewski-Barlem, J. G., Lunardi, V. L., \& Ramos, A. M. (2018). Fatores percebidos pelos acadêmicos de enfermagem como desencadeadores do estresse no ambiente formativo. Texto contexto-enferm, $27(1)$, e0370014.

Lipp, M. (2004). O stress no Brasil: Pesquisas avançadas: Papirus.

Lipp. M. (2008). O stress do professor. (6 $6^{\mathrm{a}}$. ed.): Papirus.

Luft, C. D. B., Sanches, S. O., Mazo, G. Z., \& Andrade, A. (2007). Versão brasileira da Escala de Estresse Percebido: tradução e validação para idosos. Rev Saúde Pública, 41(4), 606-615. https://doi.org/10.1590/S0034-89102007000400015.

Margis, R., Picon, P., Cosner, A. F., \& Silveira, R. D. O. (2003). Relação entre estressores, estresse e ansiedade. R. Psiquiatr. RS, 25 (supl 1), 65-74.

Mussi, F. C., Pires, C. G. S., Carneiro, L. S., Costa, A. L. S., Ribeiro, F. M. S. S., \& Santos, A. F. (2019). Comparison of stress in freshman and senior nursing students. Rev Esc Enferm USP, 53, e03431. http://dx.doi.org/10.1590/S1980-220X2017023503431

Oliveira, A. L., Godoy, M. M. C., \& Fogaça, F. F. S. (2019). Desemprego, resiliência e reinserção no mercado de trabalho. Revista Brasileira de Gestão e Desenvolvimento Regional, 15(7), 301-315. https://rbgdr.net/revista/index.php/rbgdr/article/view/5276/891 
Research, Society and Development, v. 10, n. 7, e36810716584, 2021 (CC BY 4.0) | ISSN 2525-3409 | DOI: http://dx.doi.org/10.33448/rsd-v10i7.16584

Preto, V. A., Souza, A. L. T. De., Sousa, B. de O. P., Fernandes, J. M., Pereira, S. de S., \& Cardoso, L. (2020). Preditores de estresse recente em universitários de enfermagem. Research, Society and Development, 9(3), e37932371. https://doi.org/10.33448/rsd-v9i3.2371

Ribeiro, R. M., Bragiola, J. V. B., Eid, L. P., \& Pompeo, D. A. (2020). Impacto da autoestima e dos fatores sociodemográficos na auto eficácia de estudantes de graduação em enfermagem. Texto \& Contexto Enfermagem, 29, e20180429. http://dx.doi.org/10.1590/1980-265X-TCE-2018-0429

Rocha, T. P. D. O., Matos, M. S., Correa, F. B., Silva, C. O., \& Burla, R. D. S. (2018). Anatomofisiologia do estresse e o processo de adoecimento. Revista Científica da FMC, 13(2).

Santos, E. O., Loiola, E., \& Santos, S. O. (2018). Expansão do ensino superior privado na perspectiva de desenvolvimento regional no Brasil: a realidade do sul maranhense. Cad. Pesq, 25(2), 91-107

Silva, D. A. (2019). A autoestima e o comportamento suicida em estudantes universitários: uma revisão da literatura. Revista Eletrônica Acervo Saúde, (23), e422. https://doi.org/10.25248/reas.e422.2019

Silva, D. A., \& Marcolan, J. F. (2015). Unemployment and psychological distress in nurses. Revista Brasileira de Enfermagem, 68(5), 493-500. http://dx.doi.org/10.1590/0034-7167.2015680502i

Silva, D. A., Pereira Junior, R. J., Gomes, C. F. M., \& Cardoso, J. V. (2019). Envolvimento com álcool, tabaco e outras substâncias por estudantes universitários. Revista Cuidarte, 10(2), e641. https://doi.org/10.15649/cuidarte.v10i2.641

Siqueira, J. F. (2007). A realidade contraditória e de sobrevivência do jovem trabalhador e estudante nas escolas estaduais de Porto Alegre/ RS /Brasil. Rexe, 1(1), 227-244. https://dialnet.unirioja.es/ejemplar/178799

Souza, D. C. C., \& Vazquez, D. A. (2015). Expectativas de jovens do ensino médio público em relação ao estudo e ao trabalho. Educ. Pesqui., 41(2), 409-426.

Souza, R. M. (2017). Notas sobre trabalhadores que voltam a estudar em curso superior privado. Revista Café com Sociologia, 6(2), 7-19.

Vieira, I., \& Russo, J. A. (2019). Burnout e estresse: entre medicalização e psicologização. Physis: Revista de Saúde Coletiva, 29(2), e290206. https://doi.org/10.1590/S0103-73312019290206 